\title{
Weak self-association of cytochrome $c$ peroxidase molecules observed by paramagnetic NMR
}

\author{
Jesika Schilder $^{1} \cdot$ Marcellus Ubbink $^{1}$
}

Received: 22 March 2016/ Accepted: 3 May 2016/Published online: 28 May 2016

(c) The Author(s) 2016. This article is published with open access at Springerlink.com

\begin{abstract}
There is growing experimental evidence that many proteins exhibit a tendency for (ultra)weak homo- or hetero- oligomerization interactions. With the development of paramagnetic relaxation enhancement NMR spectroscopy it has become possible to characterize weak complexes experimentally and even detect complexes with affinities in the $1-25 \mathrm{mM}$ range. We present evidence for a weak complex between cytochrome $c$ peroxidase $(\mathrm{CcP})$ molecules. In a previous study, we attached nitroxide based spin labels at three positions on $\mathrm{CcP}$ with the intent of observing intramolecular PRE effects. However, several intermolecular PRE effects were also observed suggesting a weak self-association between $\mathrm{CcP}$ molecules. The $\mathrm{CcP}-$ $\mathrm{CcP}$ complex was characterized using paramagnetic NMR and protein docking. The interaction occurs between the
\end{abstract}

surface that is also part of the stereo-specific binding site for its physiological partner, cytochrome $c(C c)$, and several small, positively charged patches on the "back" of $\mathrm{CcP}$. The $\mathrm{CcP}-\mathrm{CcP}$ complex is not a stereo-specific complex. It is a dynamic ensemble of orientations, characteristic of an encounter state. The contact areas resemble those observed for $\mathrm{CcP}$ molecules in crystals. The $\mathrm{CcP}-$ $\mathrm{CcP}$ complex formation competes with that of the $\mathrm{CcP}-\mathrm{Cc}$ complex. However, the affinity for $\mathrm{Cc}$ is much larger and thus it is expected that, under physiological conditions, auto-inhibition will be limited.

Graphical Abstract A weak self-association between cytochrome $c$ peroxidase molecules was characterized using paramagnetic NMR.

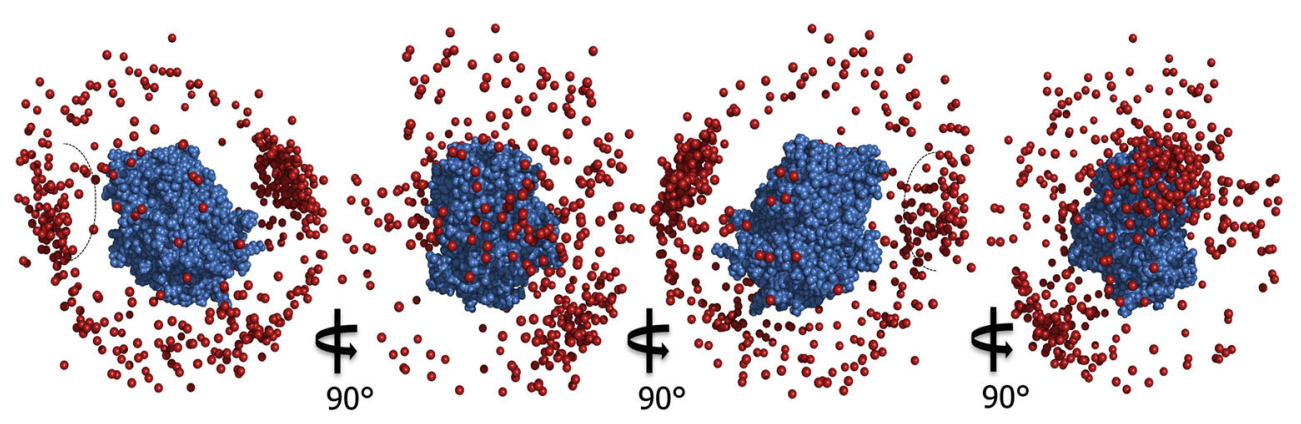

Electronic supplementary material The online version of this article (doi:10.1007/s10858-016-0035-z) contains supplementary material, which is available to authorized users.

Marcellus Ubbink

m.ubbink@chem.leidenuniv.nl

1 Gorlaeus Laboratories, Leiden Institute of Chemistry, Leiden

University, Einsteinweg 55, 2333 CC Leiden, The

Netherlands
Keywords Cytochrome $c$. Ultra-weak interactions · Paramagnetic relaxation enhancement · Ensemble docking · Electron transfer 


\section{Introduction}

Decades of research on protein-protein interactions have revealed valuable information about the structure and function of many protein complexes. While the majority of this work has focused on proteins that form strong and often highly specific interactions, the importance of the complexes of weakly associated proteins is becoming increasingly clear. These transient complexes are commonly formed to counter-balance the biological need for a specific interaction with the necessity of maintaining a high turnover rate (Schilder and Ubbink 2013). Transient complexes also include ultra-weak interactions, defined as having a dissociation constant $\left(K_{\mathrm{D}}\right)$ in the millimolar range, (Tang et al. 2008a, b; Vaynberg and Qin 2006) that are known to drive self-assembly of higher order homogeneous architectures like crystals, viral capsids and amyloid fibrils (Fawzi et al. 2007; Garcia-Ruiz 2003; Zlotnick 2005). They also play an important role in an array of cellular processes including rapid assembly/disassembly, protein maturation, reversible cell adhesion and cell signalling (Vaynberg and Qin 2006). While strongly associated protein complexes often consist of low energy, specific states that are easily isolated and studied, weakly associated protein complexes often also occupy higher energy conformations, such as the encounter state (Kleckner and Foster 2011; Ubbink 2009). These conformations are lowly populated, transient and cannot be isolated, making them practically invisible to conventional structural biology techniques (Clore 2011).

The development of new paramagnetic nuclear magnetic resonance spectroscopy (NMR) techniques has enabled characterization of these transient states in populations as low as $0.5 \%$ (Keizers and Ubbink 2011). Paramagnetic relaxation enhancement (PRE) is particularly well-suited to studying lowly populated states, as the dipolar interaction with the unpaired electron is very strong and the strength of the PRE falls off with the sixth power of the distance between the paramagnetic centre and observed nucleus, making PRE extremely distance dependent (Iwahara and Clore 2006; Tang et al. 2006; Volkov et al. 2006). PRE has been successfully used to characterize several encounter complexes (Fawzi et al. 2010; Hiruma et al. 2013; Scanu et al. 2012; Suh et al. 2007; Volkov et al. 2006; Xu et al. 2008, 2009) including the cytochrome $c$ (Cc)-cytochrome $c$ peroxidase (CcP) encounter complex (Bashir et al. 2010; Schilder et al. 2014; Van de Water et al. 2014; Volkov et al. 2006, 2010a). It has also been used to study proteinDNA complexes (Iwahara and Clore 2006; Iwahara et al. 2004, 2006) as well as large scale domain motions (Henzler-Wildman et al. 2007; Tang et al. 2007) and transient structures in unfolded and intrinsically disordered proteins
(Bertoncini et al. 2005; Dedmon et al. 2005; Gillespie and Shortle 1997a, b; Shortle and Ackerman 2001).

In 2008, PRE was used for the first time to visualize ultra-weak self-association $\left(K_{\mathrm{D}} \geq 15 \mathrm{mM}\right)$ between histidine-containing phosphocarrier protein (HPr) molecules as these dimers could not be observed by other techniques. Paramagnetic EDTA- $\mathrm{Mn}^{2+}$ tags were placed at three positions on the surface of unlabelled HPr. This protein was mixed 1:1 (300 $\mu \mathrm{M}$ each) with $\left[{ }^{15} \mathrm{~N}\right]$-labelled $\mathrm{HPr}$ and PRE were measured. An ultra-weak self-association was observed, representing a population of $1 \%$, which disappeared when the physiological HPr binding partner, enzyme I, was added. Furthermore, this interaction could be modulated by changes in the ionic strength or with charge mutations (Tang et al. 2008a). In the same year, PRE was used to show an ultra-weak interaction between the N-terminal extension of the HIV-1 protease precursor and the protein's active site resulting in autocleavage and maturation of the protein. The ultra-weak encounter complex $\left(K_{\mathrm{D}}=3-6 \mathrm{mM}\right)$ was found to represent $3-5 \%$ of the total population in a concentration of $200 \mu \mathrm{M}$ (Tang et al. 2008b). This technique has since been applied to several ultra-weak complexes (Johansson et al. 2014; Liu et al. 2012; Villareal et al. 2011) with $K_{\mathrm{D}}$ values as high as $25 \mathrm{mM}$ (Xing et al. 2014). Together, these studies demonstrated the use of PRE for the observing weak interactions of self-associations that are difficult to visualize with conventional methods.

In our previous work using nitroxide based spin labels to obtain intramolecular PRE data on CcP, we observed multiple unexpected PRE effects for residues further than $24 \AA$ from the spin label, the PRE limit for nitroxide radicals (Keizers and Ubbink 2011). Here, we show that these additional PRE are in fact intermolecular effects generated by a weak self-association between the $\mathrm{CcP}$ molecules. Furthermore, we have characterized this weak $\mathrm{CcP}-\mathrm{CcP}$ complex using paramagnetic NMR and ensemble docking. Until now, $\mathrm{CcP}$ had been assumed to exist as a monomer in solution. This is interesting for understanding the aggregation propensity of proteins, particularly in the crowded cellular environment. However, the potential biological relevance of a $\mathrm{CcP}-\mathrm{CcP}$ complex is unclear as the affinity of $\mathrm{CcP}$ for $\mathrm{Cc}$ is much greater than that for $\mathrm{CcP}$ and thus auto-inhibition is unlikely under physiological conditions.

\section{Materials and methods}

\section{Protein sample preparation}

Yeast $\left[{ }^{15} \mathrm{~N},{ }^{2} \mathrm{H}\right]-$ or $\left[{ }^{15} \mathrm{~N},{ }^{13} \mathrm{C}\right]$-labelled $\mathrm{CcP}$ C128A with MSKT as the first four $\mathrm{N}$-terminal residues was expressed 
and purified as published previously (Morar et al. 1999; Pollock et al. 1998; Schilder et al. 2014). The same CcP construct with the additional mutations N38C, N200C or T288C were used to produce unlabelled protein (Schilder et al. 2015; Volkov et al. 2006). 1-acetoxy-2,2,5,5-tetramethyl- $\delta 3$-pyrroline-3-methyl)-methanethiosulfonate (MTS) and 1-oxyl-2,2,5,5-tetramethyl-2,5-dihydropyrrol-3-ylmethyl methanethiosulfonate (MTSL) tags were obtained from Toronto Research Chemicals (Toronto, ON, Canada). The spin labels were stored as $100 \mathrm{mM}$ stocks dissolved in DMSO at $4{ }^{\circ} \mathrm{C}$ prior to use. The CcP mutants were tagged with MTS, MTSL as described previously (Schilder et al. 2014; Volkov et al. 2006). The tagging efficiency was determined by mass spectroscopy to be essentially $100 \%$. Yeast iso1-Cc WT was expressed and purified according to published procedures (Morar et al. 1999; Pollock et al. 1998).

\section{NMR spectroscopy}

All NMR samples contained $20 \mathrm{mM} \mathrm{NaPi}, 100 \mathrm{mM} \mathrm{NaCl}$, $6 \% \mathrm{D}_{2} \mathrm{O}, \mathrm{pH} 6.0$. The $\mathrm{pH}$ of the samples was adjusted to $6.00 \pm 0.05$, with small aliquots of $0.5 \mathrm{M} \mathrm{HCl}$ or $\mathrm{NaOH}$. To determine the optimal CcP concentration, $2 \mathrm{D}\left[{ }^{15} \mathrm{~N},{ }^{1} \mathrm{H}\right]$ TROSY-HSQC (Pervushin et al. 1997) spectra were obtained with 1024 and 128 complex points in the direct and indirect dimensions, respectively, on $400-800 \mu \mathrm{M}$ double labelled $\left[{ }^{15} \mathrm{~N},{ }^{13} \mathrm{C}\right] \mathrm{CcP}$ samples at $293 \mathrm{~K}$. Measurements were performed at ${ }^{1} \mathrm{H}$ Larmor frequencies of $600 \mathrm{MHz}$ on a Bruker Avance III spectrometer equipped with a TCI-Z-GRAD CryoProbe (Bruker, Karlsruhe, Germany). The data were processed using Topspin 3.1 (Bruker, Karlsruhe, Germany).

For inter-molecular PRE measurements, NMR samples contained $200 \mu \mathrm{M} \quad\left[{ }^{15} \mathrm{~N},{ }^{2} \mathrm{H}\right]$ labelled $\mathrm{CcP} \quad \mathrm{WT}$ and $200 \mu \mathrm{M}$ unlabelled N38C, N200C or T288C CcP with either MTS or MTSL tags attached. For intra-molecular PRE measurements, NMR samples contained $400 \mu \mathrm{M}$ of $\left[{ }^{15} \mathrm{~N},{ }^{2} \mathrm{H}\right]$-labelled tagged mutants. For intra-molecular PRE measurements in the presence of $\mathrm{Cc}, 400 \mu \mathrm{M}$ unlabelled WT Cc was also present. 2D BEST-TROSY-HSQC experiments (Lescop et al. 2007) were recorded on a Bruker AVIII HD spectrometer equipped with a ${ }^{1} \mathrm{H}\left[{ }^{13} \mathrm{C} /{ }^{15} \mathrm{~N}\right]$ TCI-cryoprobe operating at a proton Larmor frequency of $850 \mathrm{MHz}$ at $293 \mathrm{~K}$ with 1024 and 100 complex points in the ${ }^{1} \mathrm{H}$ and ${ }^{15} \mathrm{~N}$ dimensions, respectively. The data were processed using Topspin 3.2 (Bruker, Karlsruhe, Germany). All NMR data were analyzed using CCPN Analysis 2.1.5 (Vranken et al. 2005). The backbone resonance assignment for $\mathrm{CcP}$ were taken from (Schilder et al. 2014).

\section{PRE analysis}

The intensity ratio of the amide resonances in the spectra of the paramagnetic (MTSL) and diamagnetic (MTS) samples $\left(I_{\text {para }} / I_{\text {dia }}\right)$ was calculated and normalized as described previously (Bashir et al. 2010). The paramagnetic contribution to the transverse relaxation rate, $R_{2, \text { para }}$, was calculated as reported previously (Bashir et al. 2010; Battiste and Wagner 2000; Schilder et al. 2014). For the amide peaks that disappeared in the paramagnetic spectrum, an upper limit for $I_{\text {para }}$ was set to two standard deviations of the noise level of the spectrum (Schilder et al. 2014).

The calculated $R_{2, p a r a}$ values were then converted into distances as described previously (Eq. 1) (Bashir et al. 2010):

$r=\sqrt[6]{\frac{f_{\text {bound }}}{R_{2, \text { para }}} \frac{\gamma_{H}^{2} g_{e}^{2} \beta^{2} \mu_{0}^{2}(S+1) S}{240 \pi^{2}}\left(4 \tau_{c}+\frac{3 \tau_{c}}{1+\omega_{H}^{2} \tau_{c}^{2}}\right)}$

where $r$ is the distance between the oxygen atom of the spin label nitroxide and a given amide proton, $f_{\text {bound }}$ is the fraction of observed protein sample bound to the paramagnetic protein (estimated at 0.40 ), $\gamma_{\mathrm{H}}$ is the proton gyromagnetic ratio, $g_{e}$ is the electronic g-factor, $\beta$ is the Bohr magneton, $\mu_{0}$ is the vacuum permeability, $S$ is the spin quantum number for the spin label $(1 / 2)$ and $\omega_{\mathrm{H}}$ is the proton Larmor frequency in rad/s (Battiste and Wagner 2000; Bertini et al. 1996). $\tau_{\mathrm{c}}$ is the correlation time of the vector connecting the radical and the observed nucleus. The $\tau_{\mathrm{c}}$ is expected to be dominated by the rotational correlation time of the $\mathrm{CcP}-\mathrm{CcP}$ complex, which was estimated to be $45 \mathrm{~ns}$ (Bernado et al. 2002). The calculated distances were divided into three classes: strongly affected residues for which the peaks had been completely broadened out in the paramagnetic spectrum and only an upper limit could be calculated (class I), affected residues for which the peaks were visible in the paramagnetic spectrum (error margins were set to at least $\pm 3 \AA$ to account for experimental error, class II) and residues that were too far away from the spin label to experience significant PRE, so only a lower limit could be calculated (class III) (Bashir et al. 2010; Schilder et al. 2014). We prefer converting PREs to distances rather than to dock directly with PREs because it makes the relation between PRE, $\tau_{\mathrm{c}}$ and $f_{\text {bound }}$ explicit. In graphical evaluations of back-calculated data, comparing distances puts the emphasis on the most important class II restraints, whereas plotting PREs emphasizes the less defined class I restraints.

\section{Ensemble docking}

The coordinates for $\mathrm{CcP}$ were obtained from the crystal structure of the complex with Cc, PDB 2PCC (Pelletier and Kraut 1992). The docking of $\mathrm{CcP}$ to $\mathrm{CcP}$ was driven by a 


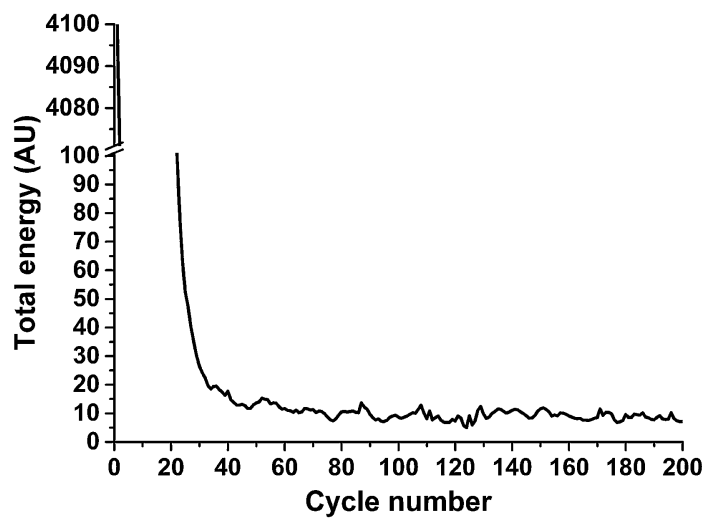

Fig. 1 Total energy (in arbitrary units) during a single approach of five copies of $\mathrm{CcP}$ with spin label tags on a single untagged $\mathrm{CcP}$. An approach consists of 200 cycles with the lowest energy structure being saved

set of distance restraints derived from inter-molecular PRE data using Xplor-NIH version 2.34 (Schwieters et al. 2003, 2006). This was done using an ensemble of four spin label conformers, the orientations of which were fixed in experimentally determined orientations published previously (Schilder et al. 2015). One to eight copies of CcP with spin label tags were docked to untagged $\mathrm{CcP}$ using rigid body dynamics with van der Waals repel forces and the distance restraints contributing to the total energy. The distance between the haem iron atoms of the CcP molecules was restrained to $20-60 \AA$. Docking was repeated from random starting positions using 100 approaches of 200 cycles each (Fig. 1) in which the lowest energy structure of each approach was saved, resulting in 100 structures. One cycle consisted of 1000 steps of 0.4 ps in the dynamics mode of Xplor-NIH.

The twenty lowest energy structures of $\mathrm{CcP}$ in the ensemble were analyzed to determine the optimal number of copies of $\mathrm{CcP}$ required. With more than five copies of unlabelled $\mathrm{CcP}$ in the ensemble, the quality of the fit to the PRE does not improve. Then the docking was repeated for 1000 approaches of 200 cycles each, resulting in 1000 structures of which the 100 lowest energy structures were used to build the ensemble. The back-calculated distances were obtained by taking the $\mathrm{r}^{-6}$ average over the four spin label rotamers at each position followed by a linear averaging of the values for the 20 or 100 lowest energy ensemble solutions. The fit between the observed (dis ${ }^{\mathrm{obs}}$ ) and back-calculated ( dis $^{\text {calc }}$ ) distances for the class II restraints was evaluated using a Q-factor according to (Eq. 2):(Bashir et al. 2010)

$Q=\sqrt{\frac{\sum_{i}\left(d i s_{i}^{o b s}-d i s_{i}^{c a l c}\right)^{2}}{\sum_{i}\left(d i s_{i}^{o b s}+d i s_{i}^{c a l c}\right)^{2}}}$
Note that in this definition, the denominator is the sum of the observed and calculated distances. The average violation (AV) was determined as described previously, (Schilder et al. 2015) by averaging the difference between the experimental and back-calculated distances; for distances with only an upper (class I) or lower boundary (class III), back-calculated distances that fell inside of those boundaries were not considered violations.

\section{Results and discussion}

The first evidence for weak self-association between CcP molecules appeared while optimizing the $\mathrm{CcP}$ concentration for the backbone amide resonance assignment of $\mathrm{CcP}$ (Schilder et al. 2014). Previous NMR studies on the complex between $\mathrm{CcP}$ and $\mathrm{Cc}$ were done using a 1:1 ratio at $300 \mu \mathrm{M}$ of each protein (Bashir et al. 2010; Volkov et al. 2006, 2010b). Cc is remarkably soluble $(\sim 100 \mathrm{mg} /$ $\mathrm{mL}$ ) (Volkov et al. 2011) so the NMR sample concentration was previously limited by the solubility of CcP. However, in our hands, our $\mathrm{CcP}$ mutants are stable at much higher concentrations. It was hoped that indeed higher concentrations of $\mathrm{CcP}$ could be used during NMR experiments in order to take advantage of the increased signal intensity that would provide. To determine the optimal CcP concentration for the NMR samples, $2 \mathrm{D}\left[{ }^{15} \mathrm{~N},{ }^{1} \mathrm{H}\right] \mathrm{TROSY}$ -

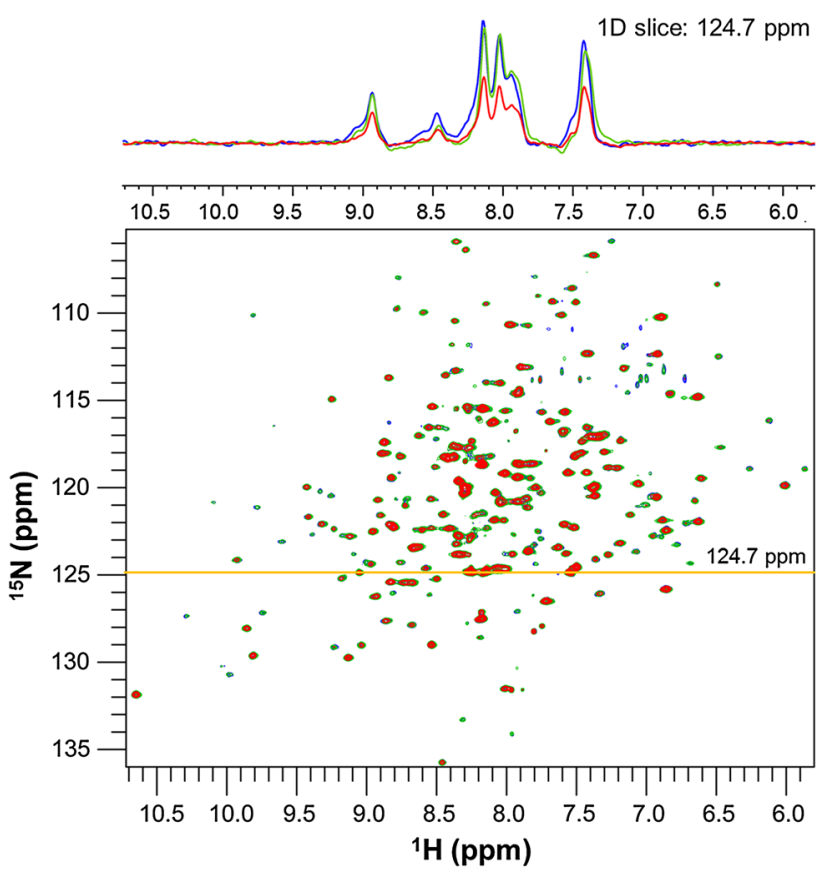

Fig. $22 \mathrm{D} \quad\left[{ }^{15} \mathrm{~N},{ }^{1} \mathrm{H}\right]$ TROSY-HSQC spectra of $400 \mu \mathrm{M}$ (blue), $600 \mu \mathrm{M}$ (green) or $800 \mu \mathrm{M}$ (red) double labelled $\left[{ }^{13} \mathrm{C},{ }^{15} \mathrm{~N}\right] \mathrm{CcP}$ C128A with 1D overlay (above) showing relative ${ }^{1} \mathrm{H}$ peak intensities at $124.7 \mathrm{ppm}$ in the ${ }^{15} \mathrm{~N}$ dimension (yellow line). Experiments were performed at $20{ }^{\circ} \mathrm{C}$ in $20 \mathrm{mM} \mathrm{NaPi}, 100 \mathrm{mM} \mathrm{NaCl}, \mathrm{pH} 6.0$ 
HSQC spectra were obtained for samples containing 400-800 $\mu \mathrm{M}$ CcP (Fig. 2).

In a sample that does not aggregate, the signal-to-noise ratio should be proportional to the sample concentration. However, although no visible aggregation was observed in the sample, the 1D traces of the spectra showed little or no peak intensity increase when going from 400 to $600 \mu \mathrm{M}$ and a large decrease in peak intensity when the sample concentration was further increased to $800 \mu \mathrm{M}$ (Fig. 2). This suggests that indeed aggregation of $\mathrm{CcP}$ was occurring in the sample. When proteins aggregate, the intensity increase at higher concentrations is counteracted by enhanced nuclear relaxation, resulting in intensity loss due to the larger rotational correlation time of the aggregate, as compared to the monomeric state of the protein. Weak selfassociation has been seen in other proteins at concentrations 200-500 $\mu \mathrm{M}$ (Johansson et al. 2014; Liu et al. 2012; Tang et al. 2008a, b).

Further evidence for a weak $\mathrm{CcP}-\mathrm{CcP}$ interaction was observed during a study on the use of PRE for CcP-Cc complex structure determination (Schilder et al. 2015). We attached the spin label MTSL at three positions on CcP around the stereo-specific binding interface for Cc (Fig. 3).

Nitroxide spin labels generate measurable PRE effects up to $24 \AA$ for a protein the size of CcP (Keizers and Ubbink 2011). Thus, no intramolecular PRE were expected beyond this limit. However, we found many PREs for residues spread across the $\mathrm{CcP}$ sequence, including for residues more than $24 \AA$ from the spin label attachment site. By converting these PREs into the distances between the nitroxide oxygen atom and the amide hydrogens of $\mathrm{CcP}$, the intra- and intermolecular PRE could be distinguished (Figure S1). Furthermore, upon the addition of Cc, the physiological binding partner for $\mathrm{CcP}$, the suspected intermolecular PRE effects were diminished (Figure S2). The dissociation constant, $K_{\mathrm{D}}$, for the interaction between $\mathrm{Cc}$ and $\mathrm{CcP}$ is $5 \mu \mathrm{M}$, (Schilder et al. 2014; Volkov et al. 2009; Worrall et al. 2001) while the value for a CcP-CcP interaction would be expected to be orders of magnitude higher (Tang et al. 2008a, b; Vaynberg and Qin 2006).

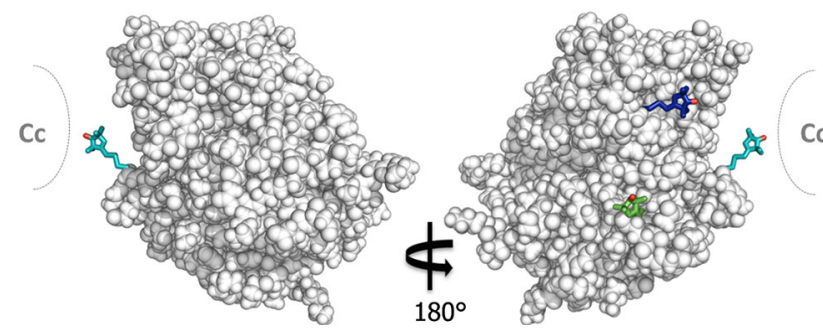

Fig. 3 Locations of spin labels attached on the surface of $\mathrm{CcP}$ at positions C38 (teal), C200 (blue) and C288 (green) showing the nitroxide oxygen atom in red (PDB-entry 2PCC) (Pelletier and Kraut 1992). The binding site of $\mathrm{Cc}$ is shown schematically
Therefore, assuming the $\mathrm{CcP}-\mathrm{CcP}$ interaction occurs via the stereo-specific binding interface for $\mathrm{Cc}$, the addition of $\mathrm{Cc}$ to the sample was expected to reduce the observed intermolecular PRE for CcP. This confirmed that ultraweak intermolecular interaction occurs between $\mathrm{CcP}$ molecules.

In order to accurately characterize this weak self-association, the PRE measurements were repeated using nonisotopically labelled $\mathrm{CcP}$ single mutants that were tagged with spin labels in a 1:1 mixture with WT $\left[{ }^{2} \mathrm{H}^{15} \mathrm{~N}\right]$ isotope labelled CcP. In this way, only intermolecular PRE effects are observed, removing any possible interference from intramolecular PRE effects. The interaction between $\mathrm{CcP}$ molecules can then be visualized by mapping the intermolecular PRE effects on a surface model of CcP (Fig. 4).

The observed PRE effects are stronger for position C38 and C200 (Fig. 4a, b, respectively) when compared to C288 (Fig. 4c). The strength of the PRE for a given residue is dependent on the distance between that residue and the

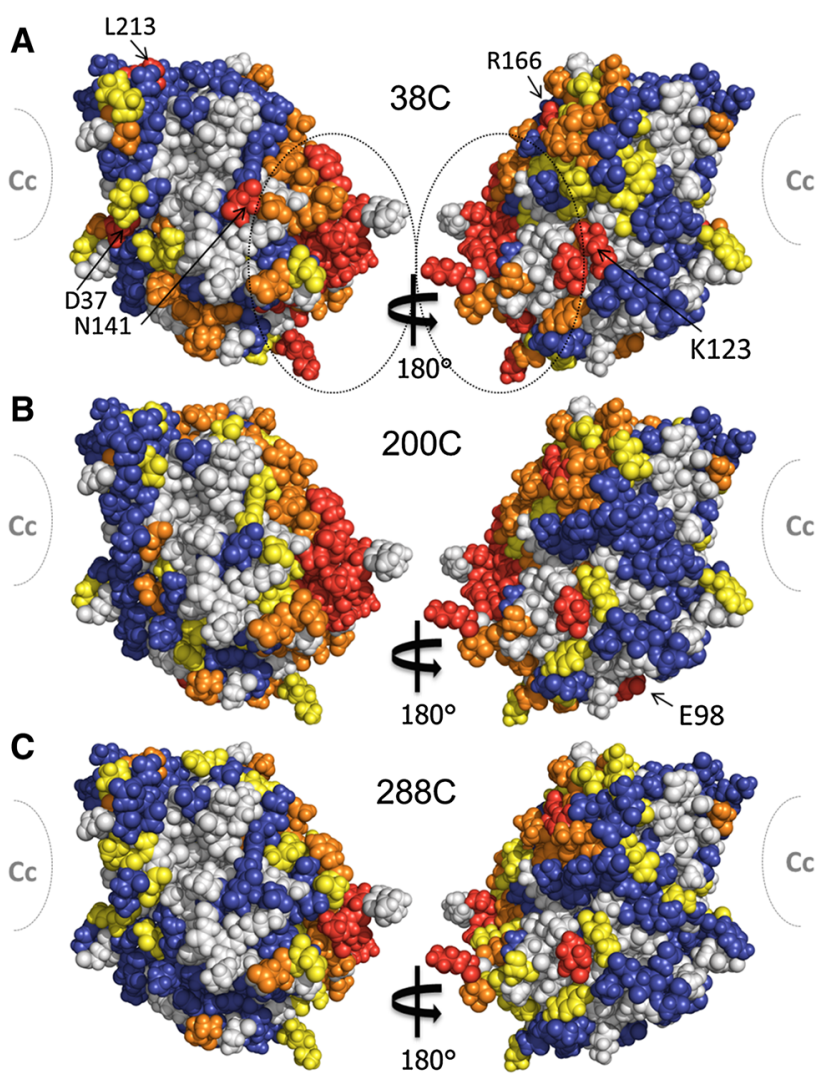

Fig. 4 Intermolecular PRE map for CcP with MTSL at positions C38 (a), C200 (b) or C288 (c). The PRE effects are colour-coded on CcP (PDB-entry 2PCC) (Pelletier and Kraut 1992). The PRE effects are mostly localized around residues 3-12, 60-63, 132, 273-278, 285 (marked with dotted oval). The location of the stereo-specific binding site for Cc is shown schematically. Residues with $R_{2}$, para $\geq 100 \mathrm{~s}^{-1}$ are red, $20 \mathrm{~s}^{-1}<\mathrm{R}_{2}$, para $<100 \mathrm{~s}^{-1}$ are orange, $5 \mathrm{~s}^{-1}$. $<\mathrm{R}_{2}$, para $<20 \mathrm{~s}^{-1}$ are yellow, $\mathrm{R}_{2}$, para $\leq 5 \mathrm{~s}^{-1}$ are blue and with no data are grey 
paramagnetic centre. Therefore, given the orientation of the $\mathrm{CcP}-\mathrm{CcP}$ complex, spin labels at some locations may be further from the main interaction site than others, resulting in this discrepancy in PRE strength. Furthermore, as the main interaction between the $\mathrm{CcP}$ molecules appears to occur between the stereo-specific binding site and the backside, placement of the spin labels close to the stereospecific binding site can interfere with the complex formation. This can result in a slightly weaker interaction for those complexes. Although the discrepancy between the PRE maps seems to be a result of the distance between the spin label attachment site and the stereo-specific binding interface (Fig. 3), the possibility of spin label interference with complex formation cannot be ruled out without further experiments.

Several of these residues (H6, K12, H60, K123, R166, K278) are partially or fully positively charged at $\mathrm{pH} 6$ and residue $\mathrm{T} 3$ is located beside $\mathrm{K} 2$, which also experienced a moderately strong PRE. Interestingly, although the majority of the remaining residues are non-polar amino acids there are also several negatively charged residues (E98, D37, D61, D132, D165). This was very unexpected for $\mathrm{CcP}$, which overall is highly negatively charged $(\mathrm{pI}=4.5)$, (Yonetani 1965) and particularly for a interaction involving the stereo-specific binding interface (Fig. 5).

The electrostatic potential map for $\mathrm{CcP}$ shows the wellknown large negative patch around the stereo-specific binding interface, (Northrup et al. 1988) which has evolved to interact with the highly positively charged Cc (Volkov et al. 2011). There are also smaller negative patches on the sides and back of $\mathrm{CcP}$, relative to the stereo-specific binding interface, but these are interspersed with small positive patches. These positive patches include most of the residues that experience large PRE (3-12, 60-63, 132, 273-278; dotted lines in Fig. 5) as might be expected for

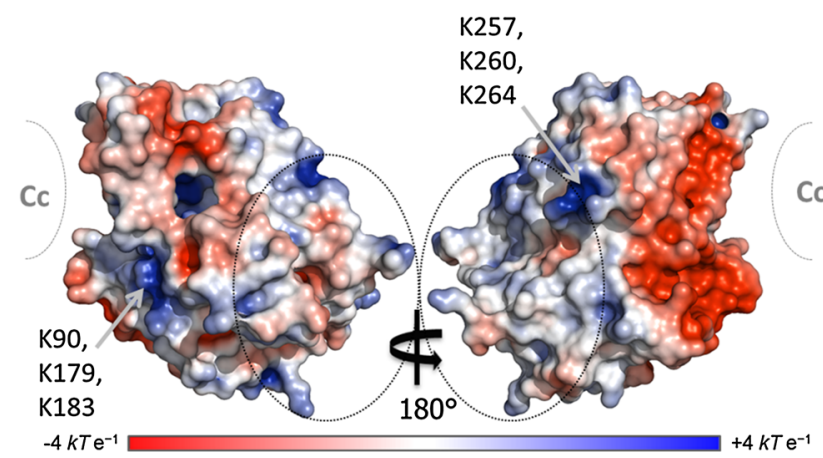

Fig. 5 Map of the electrostatic potential generated for CcP (PDBentry 2PCC) (Pelletier and Kraut 1992) The majority of the strong PRE effects are localized around residues 3-12, 60-63, 132, 273-278, 285 (dotted circles). The location of the stereo-specific binding site for $\mathrm{Cc}$ is marked in grey. The potential isocontours range from $-4 k T$ $\mathrm{e}^{-1}$ (red) to $+4 k T \mathrm{e}^{-1}$ (blue) and were calculated using APBS (Baker et al. 2001) with an ionic strength of $120 \mathrm{mM}$ at $\mathrm{pH} 6$ to match experimental conditions an interaction with the negative patch of the stereo-specific binding interface. However, the two strongest positive patches made of lysines 90, 179 and 183 and lysines 257, 260 and 264 (Fig. 5) are only moderately affected. For the first patch, residue K90 experiences moderate PRE and K183 experiences weak PRE for spin label position 38C but neither experience PRE for spin label positions $200 \mathrm{C}$ or $288 \mathrm{C}$ (no data were obtained for K179). For the second patch, although K257 experiences moderate PRE effects, K260 experiences only weak PRE for position C38 and no PRE for the other spin label positions while K264 experiences no PRE at all (Fig. 4). Therefore, although unexpected for such a highly charged protein, whose physiological interaction with $\mathrm{Cc}$ is driven predominantly by electrostatics, (Pelletier and Kraut 1992; Ulucan and Helms 2015) it appears that for the CcP self-association, specificity is driven by more than just electrostatics. Given the number of non-polar amino acids that also experienced strong PRE (Table 1), it appears that hydrophobic interactions are also playing a role, although no obvious hydrophobic interaction patch was identified.

To visualize the $\mathrm{CcP}-\mathrm{CcP}$ complex, ensemble docking was employed. Modelling of weakly interacting, highly dynamic complexes generally requires an ensemble of structures to fit the observed data (Schilder and Ubbink 2013). Such an ensemble can be created by simultaneous docking of multiple copies of one of the proteins on the other driven by the experimental PREs as restraints. During the docking process experimental parameters are compared with the back-calculated ones that are averaged over all the copies of the docked proteins (Tang et al. 2006). The use of the PRE as docking restraints requires information about the fraction bound in the complex $\left(f_{\text {bound }}\right)$ as well as the rotational correlation time $\left(\tau_{\mathrm{c}}\right)_{\text {. }}$ for the complex (see "Materials and methods" section), neither of which are known. These values are linked because both are (nearly) proportional to $r^{6}$, where $r$ is the PRE derived distance between the spin label radical and the observed nucleus. We first estimated $\tau_{\text {c- }}$ for the whole complex to be $45 \mathrm{~ns}$, which is approximately twice the value predicted for a single CcP of 20 ns (Bernado et al. 2002). Then, using this value, we estimated a fraction bound of 0.4 by establishing the lowest fraction at which the quality of fit to the experimental PREs as judged by the total docking energy no longer decreases (Figure S3). The fraction is unlikely to be larger because then larger effects on line broadening would have been expected. A fraction bound of 0.4 results in an estimated $K_{\mathrm{D}}$ of $360 \mu \mathrm{M}$ for the CcP-CcP complex. This is approximately 70 times weaker than that of the physiological $\mathrm{Cc}-\mathrm{CcP}$ complex, $5 \mu \mathrm{M}$, (Schilder et al. 2014; Volkov et al. 2009; Worrall et al. 2001) and is in agreement with the observation that addition of $\mathrm{Cc}$ to the sample diminishes the $\mathrm{CcP}-\mathrm{CcP}$ interaction. 
Table 1 CcP residues strongly affected $\left(\mathrm{R}_{2}\right.$ para $\left.\geq 100 \mathrm{~s}^{-1}\right)$ by intermolecular PRE caused by spin labels attached at positions C38, C200 or C288

\begin{tabular}{ll}
\hline Position & CcP Residues \\
\hline C38 & T3, L4, V5, H6, V7, A8, V10, K12, D37, H60, N62, K123, D132, N141, D165, L213, G273, I274, T275, F276, K278, I285 \\
C200 & T3, L4, V5, H6, V7, H60, D61, N62, T63, E98, D165, R166, G189, G273, I274, T275, F276, K278, I285 \\
C288 & T3, L4, V5, D165, R166, K278, I285 \\
\hline
\end{tabular}

The experimental PREs were measured in a sample containing $200 \mu \mathrm{L}\left[{ }^{15} \mathrm{~N},{ }^{2} \mathrm{H}\right]$-labelled $\mathrm{CcP}$ and $200 \mu \mathrm{L} \mathrm{CcP}$ tagged with MTS(L). These residues are coloured red in Fig. 4

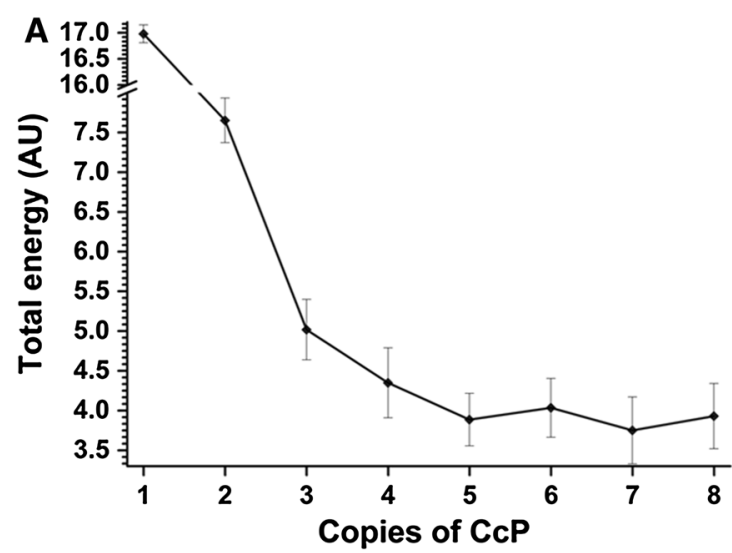

Fig. 6 Results for docking of one or multiple copies of $\mathrm{CcP}$ with spin label tags on a single untagged $\mathrm{CcP}$ based on experimental PREs, assuming a $\tau_{\mathrm{c}}$ of $45 \mathrm{~ns}$ and a fraction bound of 0.4. a Shows the total energy of the ensemble compared to the number of copies of $\mathrm{CcP}$ with spin label tags docked on a single untagged $\mathrm{CcP}$ and $\mathbf{b}$ the $\mathrm{Q}$ factor for

We found that docking five copies of spin label tagged $\mathrm{CcP}$ on a single copy of untagged $\mathrm{CcP}$ fitted the PRE data well and increasing the number of copies of spin label tagged $\mathrm{CcP}$ did not greatly improve the results (Fig. 6). Therefore, protein docking was done using five copies of $\mathrm{CcP}$ and 1000 ensembles were generated. From these, the 100 lowest energy solutions were used to back-calculate the distances between the paramagnetic centre and the $\mathrm{CcP}$ amide protons and compared to the experimentally observed distances (Fig. 7). The experimental and backpredicted values are shown in red and blue lines, respectively. Note that the back-predicted distances show considerable variation (light blue bars represent $1 \mathrm{SD}$ from the mean). Clearly, very different sets of 5 structures can satisfy the data. When this variation is taken into account along with the experimental error margins (in grey), only a few residues show significant differences between the predicted and experimental distances. These include residues 123 and 213 for positon C38, 7, 236 and 285 for positon C200 and 4 and 189 for positon C288.

The fit between the experimental and back-predicted distances was expressed using both a Q-factor (Eq. 2) and

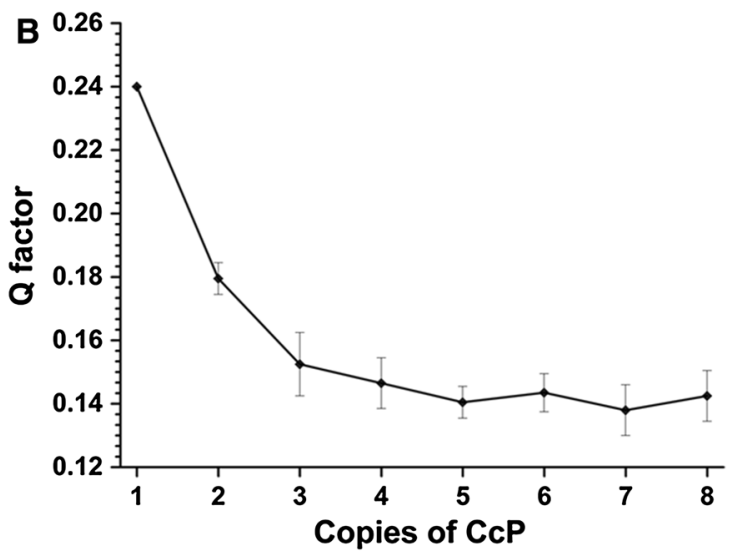

the calculated distances between the MTSL oxygen atoms and the unlabelled $\mathrm{CcP}$ amide protons. The total energy is given in arbitrary units and the error bars represent \pm 1 standard deviation for the average calculated distance from the 20 lowest-energy solutions of 100 ensembles

the average violation (AV). The Q-factor can only be calculated for the class II restraints, distances between 15.3 and $23.7 \AA$ with both an upper and lower bound (as described in "Materials and methods" section). The AV calculation can also be used for class I and III restraints, which only have an upper or lower boundary, respectively, as back-calculated distances that fall inside of those boundaries are not considered violations. The average $\mathrm{Q}$ factor is 0.11 and the average violation (AV) is $0.60 \AA$ for the data of the three spin label positions. (Table 2).

The model of the CcP-CcP complex based on the 100 ensembles of each five copies is shown in Fig. 8 as one $\mathrm{CcP}$ molecule in space-fill representation and the other $\mathrm{CcP}$ molecules shown as red spheres representing their haem irons. In line with the PRE map (Fig. 4), spin label tagged $\mathrm{CcP}$ samples a broad area of the untagged $\mathrm{CcP}$ surface. The majority of the conformations are clustered around the "back" of $\mathrm{CcP}$ in an area bordered by several of the residues that showed strong PRE effects (Fig. 8-cluster 1): residues 60-61, 123, 165-166, 273-278 and 285 (Table 1). At the "back" of CcP, there is also a second cluster of conformations (Fig. 8-cluster 2), bordered by several 

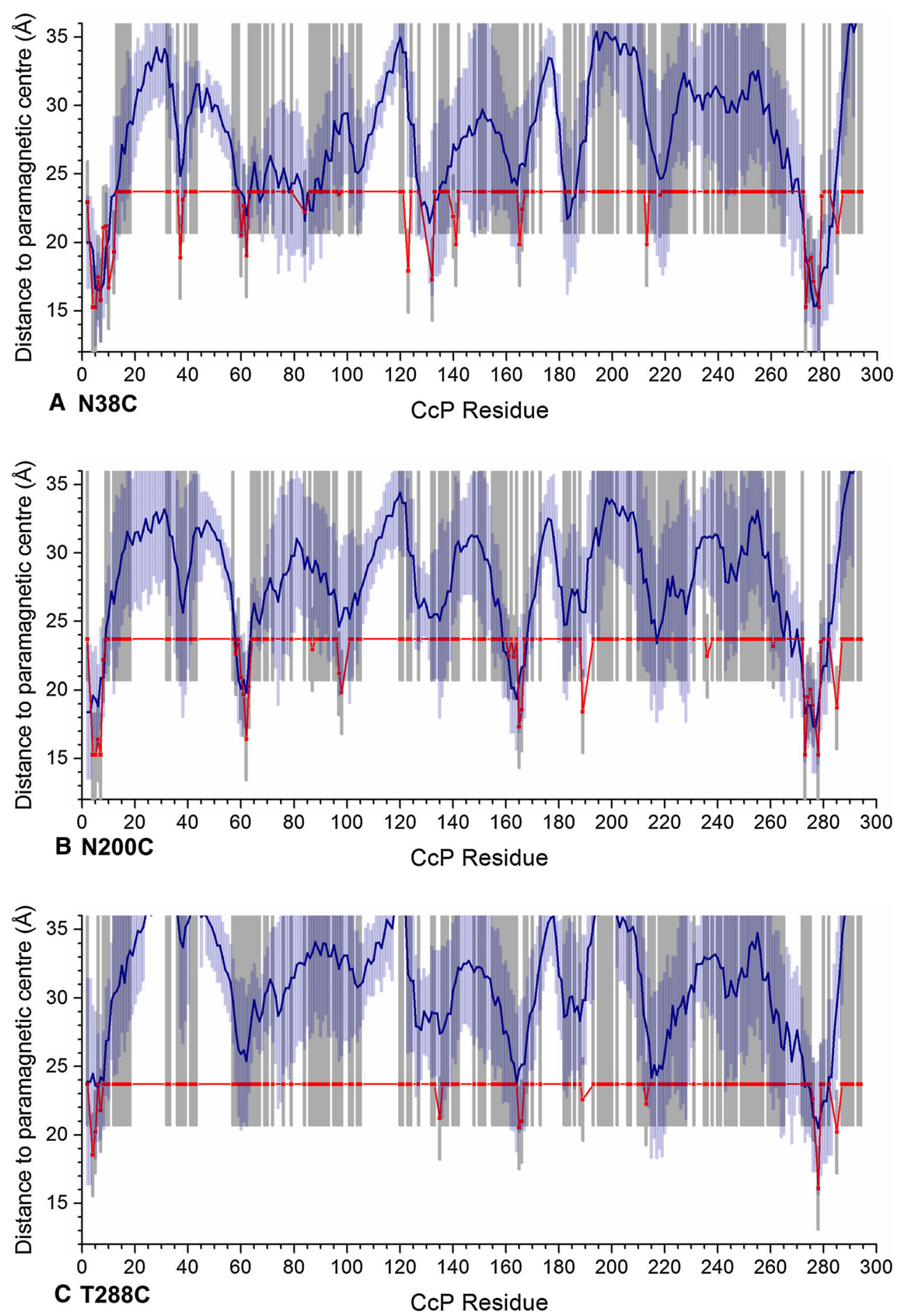

Fig. 7 Experimental and averaged back-calculated distances between CcP C128A amide protons and the paramagnetic centre in MTSL attached to $\mathrm{C} 38$ (a), $\mathrm{C} 200$ (b) or $\mathrm{C} 288$ (c) on CcP plotted against the residue number. The red line represents the experimental distances with errors in grey bars. The averaged distances over the best 100

more residues that experienced strong PRE, 62-63, 132 and 141. Residues 3-12 sit between these two clusters and so likely experience effects from conformations in both ensemble $(\mathrm{n}=5)$ solutions from 1000 dockings are shown as a blue line with a spread of one standard deviation shown in light blue bars. The experimental data were extrapolated to $100 \%$ bound $\mathrm{CcP}$, assuming a $\tau_{\mathrm{c}}$ of $45 \mathrm{~ns}$ and a fraction bound of $40 \%$

clusters. From cluster 2, there is a string of low-energy conformations that extends to a cluster at the "front" of CcP (Fig. 8-cluster 3), near the stereo-specific binding 
Table 2 Q-factors and average violations (AV) for the fit of the back-calculated to the experimental distances derived from intermolecular PRE between $\mathrm{CcP}$ amide protons and the paramagnetic centre in MTSL at positions C38, C200 or C288 for the best 100 ensemble $(n=5)$ solutions out of 1000 dockings

\begin{tabular}{llll}
\hline Position & C38 & C200 & C288 \\
\hline AV (̊) & 0.76 & 0.68 & 0.35 \\
Q-factor & 0.10 & 0.095 & 0.11 \\
\hline
\end{tabular}

site for Cc. Along this string, several more residues that experienced strong PRE are found including residues 98, 132, 37 and 213 (the latter two being located close to the stereo-specific binding site. There is also a striking absence of conformations on the "sides" of CcP (Fig. 8a, c) indicating that the interaction between $\mathrm{CcP}$ molecules occurs mainly between the stereo-specific binding interface and the "back" of CcP. This agrees with the PRE map (Fig. 4) and the observation that the CcP self-association can be reduced by the addition of $\mathrm{Cc}$.

Interestingly, several of the strongly affected residues are located at the interface between two copies of $\mathrm{CcP}$ that bind head-to-tail in the X-ray crystal structure of yeast $\mathrm{CcP}$ with horse heart Cc, PDB entry 2PCB (Pelletier and Kraut 1992). In the structure, $\mathrm{CcP}$ chain $\mathrm{C}$ residues Q120, A193, T199, D224, G228, Y229 and E290 are all within $5 \AA$ of one of the following residues in $\mathrm{CcP}$ chain $\mathrm{A}$ which are strongly affected in our study: T3, V5, V7, H60, T275 or K278. The orientation of chain $\mathrm{C}$ in $2 \mathrm{PCB}$ places it within cluster 1 of the CcP locations obtained by ensemble docking (Fig. 9a, grey ribbons). Two other CcP contacts involved in the crystal packing in this structure are also shown in the figure, as grey tubes. Their contact areas are much smaller, located close to isolated patches of residues showing PRE. Similarly, in the high-resolution structure of free CcP, PDB entry 1ZBY, (Bonagura et al. 2003) one large and one small CcP-CcP interaction area are observed. The larger contact places the $\mathrm{CcP}$ molecule within cluster 1 but in an orientation different from the one in $2 \mathrm{PCB}$ (Fig. 9b, grey ribbons). The smaller contact area places a $\mathrm{CcP}$ in a location similar to one of other two seen in $2 \mathrm{PCB}$ (Fig. 9b, grey tubes).

These findings suggest that the weak interactions observed in solution are also responsible for crystal packing. It has been suggested that the tight packing within the crystal lattice can mimic the crowded intracellular environment and that these interactions may be biologically relevant (Crowley et al. 2008). In this case, any potential biological relevance for the CcP dimer is unclear but it is unlikely to interfere with electron transfer from $\mathrm{Cc}$ due to the much greater affinity for $\mathrm{Cc}$.

Finally, a note of caution should be given for the interpretation of the results from ensemble docking. Large dynamics ensembles are always under sampled by experimental data, hence the large variation in ensembles that can fit the date (Fig. 7) (Longinetti et al. 2006). Moreover, accurate conversion of the experimental PRE values into distance restraints relies on accurate values for both the $\tau_{\mathrm{c}}$ and fraction bound, which could only be estimated. It was also assumed that the fraction bound is the same for all three spin label positions, which may not be the case if the presence of the spin label is affecting complex formation. Therefore, such models of encounter complexes should be considered only as approximations of the true encounter complex ensemble. However, they help to visualize the regions that are affected most prominently and thus very likely also responsible for the dominant interactions in the complex.

\section{Conclusions}

In recent decades, advances in paramagnetic NMR techniques, and PRE in particular, have enabled the detailed characterization of transient, lowly populated states of weakly interacting protein complexes (Keizers and Ubbink
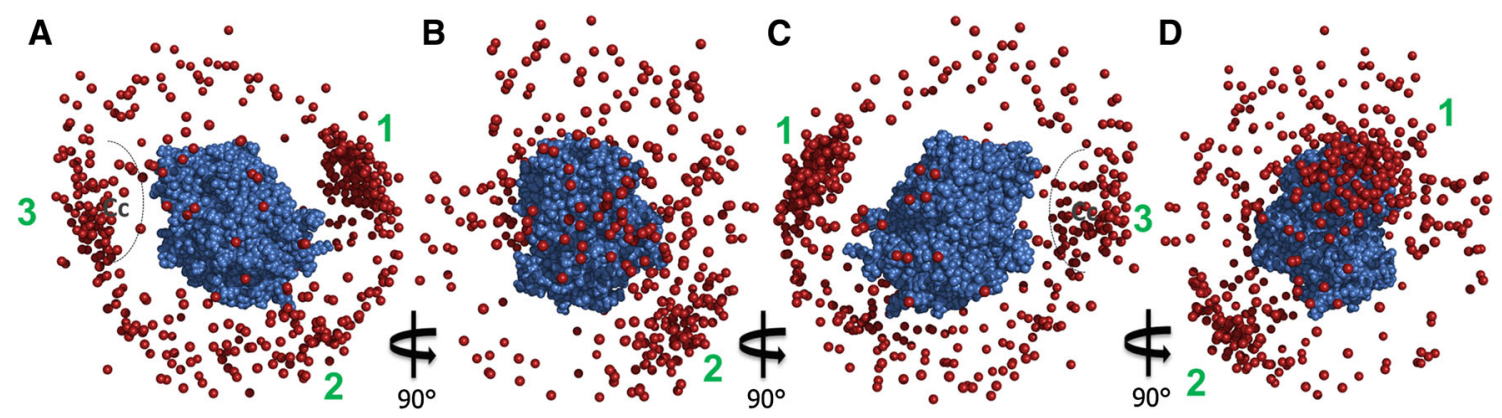

Fig. 8 The 100 lowest-energy solutions for docking an ensemble of five copies of $\mathrm{CcP}$ with spin label tags on a single untagged $\mathrm{CcP}$ driven by intermolecular PRE data. The unlabelled CcP is shown in blue spheres. For clarity, the spin label tagged $\mathrm{CcP}$ copies are represented only by their haem iron atoms (red spheres). The numbers indicate the three main clusters of $\mathrm{CcP}$ positions. The stereospecific binding site for $\mathrm{Cc}$ is indicated schematically. The docking was done using the $\mathrm{CcP}$ structure taken from PDB entry 2PCC (Pelletier and Kraut 1992) 


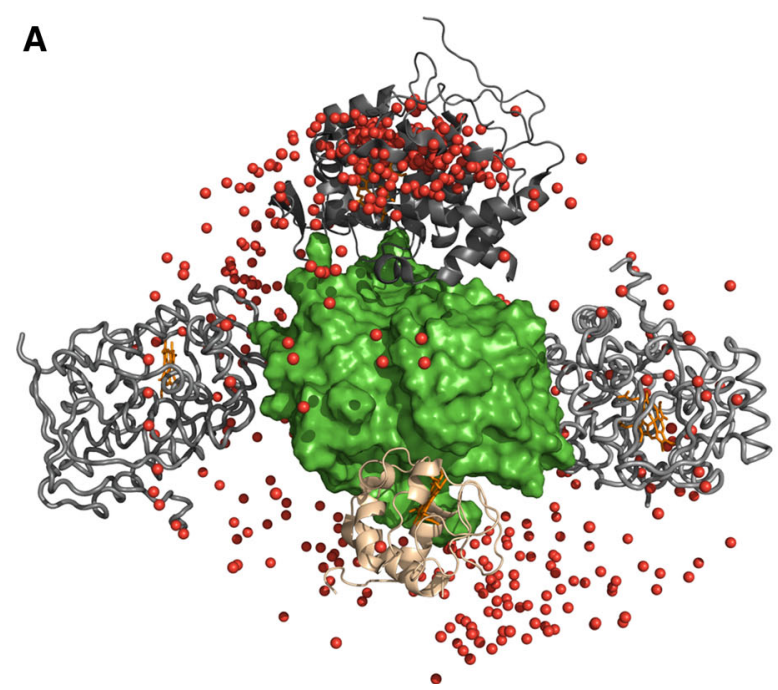

Fig. $9 \mathrm{CcP}$ crystal packing interactions. Several crystal contacts between CcP molecules in PDB entries 2PCB (Pelletier and Kraut 1992) (a) and 1ZBY (Bonagura et al. 2003) (b) are shown. The CcP (chain A) and Cc from entry 2PCC (Pelletier and Kraut 1992) are shown as green surface and beige ribbon, respectively. a The CcP molecules that contact the $\mathrm{CcP}$ chain $\mathrm{A}$ from $2 \mathrm{PCC}$ are $2 \mathrm{PCB}$ chain $\mathrm{C}$ (grey ribbon) and chains $\mathrm{G}$ and $\mathrm{I}$ (grey tubes) after alignment of $2 \mathrm{PCB}$ chains $\mathrm{A}, \mathrm{C}$ and $\mathrm{F}$, respectively, with chain $\mathrm{A}$ of $2 \mathrm{PCC}$. b The $\mathrm{CcP}$

2011). In this study, paramagnetic NMR and the PRE effect have enabled the characterization of a weak selfassociation between $\mathrm{CcP}$ molecules and provided restraints for modelling the complex using protein docking. We show that the $\mathrm{CcP}$ molecules interact with each other mainly via the stereo-specific binding interface for $\mathrm{Cc}$ and the "backside" of the protein, as if the molecules were stacking onto each other. Such weak CcP-CcP interactions resemble those seen in $\mathrm{CcP}$ crystal structures and could potentially occur within the crowded intracellular environment although it is unlikely to interfere with electron transfer from $\mathrm{Cc}$ as the affinity between $\mathrm{CcP}$ and $\mathrm{Cc}$ is much greater than that between $\mathrm{CcP}$ molecules. While the biological relevance of such dimerization is not immediately obvious, the discovery of a weak $\mathrm{CcP}$ self-association does add to a growing body of evidence showing that many proteins exhibit a tendency for (ultra-)weak homo- or hetero- oligomerization interactions (Johansson et al. 2014; Liu et al. 2012; Tang et al. 2008a, b). The existence of a $\mathrm{CcP}-\mathrm{CcP}$ interaction may also have implications for other studies on this protein, particularly when investigating weak effects, and should be taken into account when designing future experiments. Additionally, this work is a nice demonstration of the sensitivity of PRE for minor states as the level of detail the PRE data provided was much greater than that from the line broadening in the NMR spectra.

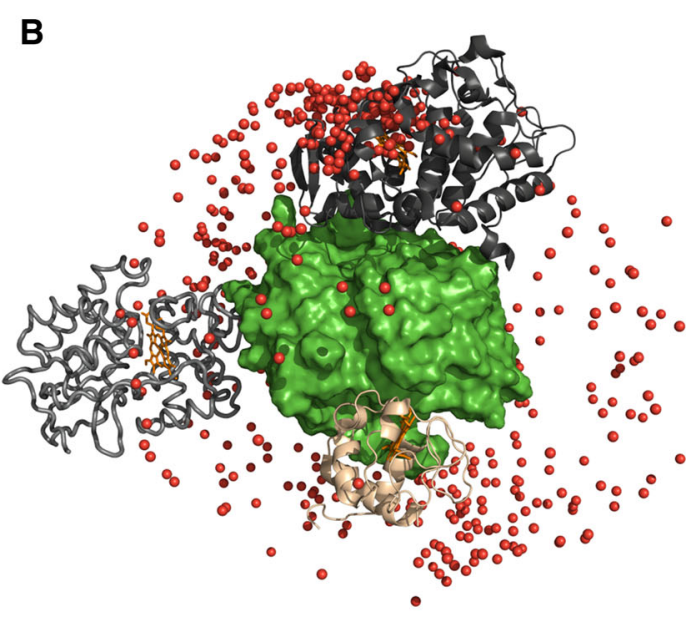

molecules that contact the $\mathrm{CcP}$ chain A from 2PCC are 1ZBY chains $\mathrm{C}$ (grey tube) and D (grey ribbon) after aligning 1ZBY chain B with chain A of 2PCC. The ribbon and tubes represent the $\mathrm{CcP}$ major and minor contact areas, respectively. Haems are shown in orange sticks. The ensemble of orientations obtained on the basis of PREs (shown in Fig. 8) is represented by the iron atoms of each $\mathrm{CcP}$ molecule, shown as red spheres

Acknowledgments We thank an anonymous referee for the suggestion to compare crystal packing orientations with those found in solution. This work was supported by the Netherlands Organization for Scientific Research (NWO), Grant 700.58.441.

Open Access This article is distributed under the terms of the Creative Commons Attribution 4.0 International License (http://crea tivecommons.org/licenses/by/4.0/), which permits unrestricted use, distribution, and reproduction in any medium, provided you give appropriate credit to the original author(s) and the source, provide a link to the Creative Commons license, and indicate if changes were made.

\section{References}

Baker NA, Sept D, Joseph S, Holst MJ, McCammon JA (2001) Electrostatics of nanosystems: application to microtubules and the ribosome. Proc Natl Acad Sci USA 98:10037-10041. doi:10. 1073/pnas.181342398

Bashir Q, Volkov AN, Ullmann GM, Ubbink M (2010) Visualization of the encounter ensemble of the transient electron transfer complex of cytochrome $c$ and cytochrome $c$ peroxidase. J Am Chem Soc 132:241-247. doi:10.1021/ja9064574

Battiste JL, Wagner G (2000) Utilization of site-directed spin labeling and high-resolution heteronuclear nuclear magnetic resonance for global fold determination of large proteins with limited nuclear overhauser effect data. Biochemistry 39:5355-5365. doi:10.1021/bi000060h

Bernado P, Garcia de la Torre J, Pons M (2002) Interpretation of ${ }^{15} \mathrm{~N}$ NMR relaxation data of globular proteins using hydrodynamic 
calculations with HYDRONMR. J Biomol NMR 23:139-150. doi:10.1023/A:1016359412284

Bertini I, Luchinat C, Aime S (1996) NMR of paramagnetic substances. Coordin Chem Rev 150:29-110. doi:10.1016/00108545(96)01242-8

Bertoncini CW, Jung YS, Fernandez CO, Hoyer W, Griesinger C, Jovin TM, Zweckstetter M (2005) Release of long-range tertiary interactions potentiates aggregation of natively unstructured alpha-synuclein. Proc Natl Acad Sci USA 102:1430-1435. doi:10.1073/pnas.0407146102

Bonagura CA et al (2003) High-resolution crystal structures and spectroscopy of native and compound I cytochrome $c$ peroxidase. Biochemistry 42:5600-5608. doi:10.1021/bi034058c

Clore GM (2011) Exploring sparsely populated states of macromolecules by diamagnetic and paramagnetic NMR relaxation. Protein Sci 20:229-246. doi:10.1002/pro.576

Crowley PB, Matias PM, Mi H, Firbank SJ, Banfield MJ, Dennison C (2008) Regulation of protein function: crystal packing interfaces and conformational dimerization. Biochemistry 47:6583-6589. doi:10.1021/bi800125h

Dedmon MM, Lindorff-Larsen K, Christodoulou J, Vendruscolo M, Dobson CM (2005) Mapping long-range interactions in alphasynuclein using spin-label NMR and ensemble molecular dynamics simulations. J Am Chem Soc 127:476-477. doi:10. $1021 / \mathrm{ja} 044834 \mathrm{j}$

Fawzi NL, Okabe Y, Yap EH, Head-Gordon T (2007) Determining the critical nucleus and mechanism of fibril elongation of the Alzheimer's A beta(1-40) peptide. J Mol Biol 365:535-550. doi:10.1016/j.jmb.2006.10.011

Fawzi NL, Doucleff M, Suh JY, Clore GM (2010) Mechanistic details of a protein-protein association pathway revealed by paramagnetic relaxation enhancement titration measurements. Proc Natl Acad Sci USA 107:1379-1384. doi:10.1073/pnas.0909370107

Garcia-Ruiz JM (2003) Nucleation of protein crystals. J Struct Biol 142:22-31. doi:10.1016/S1047-8477(03)00035-2

Gillespie JR, Shortle D (1997a) Characterization of long-range structure in the denatured state of staphylococcal nuclease. I. Paramagnetic relaxation enhancement by nitroxide spin labels. J Mol Biol 268:158-169. doi:10.1006/jmbi.1997.0954

Gillespie JR, Shortle D (1997b) Characterization of long-range structure in the denatured state of staphylococcal nuclease. II. Distance restraints from paramagnetic relaxation and calculation of an ensemble of structures. J Mol Biol 268:170-184. doi:10. 1006/jmbi.1997.0953

Henzler-Wildman KA et al (2007) Intrinsic motions along an enzymatic reaction trajectory. Nature 450:838-844. doi:10. 1038/nature 06410

Hiruma Y et al (2013) The structure of the cytochrome p450camputidaredoxin complex determined by paramagnetic NMR spectroscopy and crystallography. J Mol Biol 425:4353-4365. doi:10.1016/j.jmb.2013.07.006

Iwahara J, Clore GM (2006) Detecting transient intermediates in macromolecular binding by paramagnetic NMR. Nature 440:1227-1230. doi:10.1038/nature04673

Iwahara J, Schwieters CD, Clore GM (2004) Characterization of nonspecific protein-DNA interactions by ${ }^{1} \mathrm{H}$ paramagnetic relaxation enhancement. J Am Chem Soc 126:12800-12808. doi:10.1021/ja046246b

Iwahara J, Zweckstetter M, Clore GM (2006) NMR structural and kinetic characterization of a homeodomain diffusing and hopping on nonspecific DNA. Proc Natl Acad Sci U S A 103:15062-15067. doi:10.1073/pnas.0605868103

Johansson H et al (2014) Specific and nonspecific interactions in ultraweak protein-protein associations revealed by solvent paramagnetic relaxation enhancements. J Am Chem Soc 136:10277-10286. doi:10.1021/ja503546j
Keizers PH, Ubbink M (2011) Paramagnetic tools in protein NMR. Protein NMR spectroscopy: Practical techniques and applications. Wiley, Chichester, pp 193-219. doi:10.1002/9781119972006.ch6

Kleckner IR, Foster MP (2011) An introduction to NMR-based approaches for measuring protein dynamics. Biochim Biophys Acta 1814:942-968. doi:10.1016/j.bbapap.2010.10.012

Lescop E, Schanda P, Brutscher B (2007) A set of BEST tripleresonance experiments for time-optimized protein resonance assignment. J Magn Reson 187:163-169. doi:10.1016/j.jmr. 2007.04.002

Liu Z, Zhang WP, Xing Q, Ren XF, Liu ML, Tang C (2012) Noncovalent dimerization of ubiquitin. Angew Chem Int Ed Engl 51:469-472. doi:10.1002/anie.201106190

Longinetti M, Luchinat C, Parigi G, Sgheri L (2006) Efficient determination of the most favoured orientations of protein domains from paramagnetic NMR data. Inverse Probl 22:1485-1502. doi:10. 1088/0266-5611/22/4/019

Morar AS, Kakouras D, Young GB, Boyd J, Pielak GJ (1999) Expression of ${ }^{15} \mathrm{~N}$-labeled eukaryotic cytochrome $c$ in Escherichia coli. J Biol Inorg Chem 4:220-222. doi:10.1007/s007750050307

Northrup SH, Boles JO, Reynolds JC (1988) Brownian dynamics of cytochrome $c$ and cytochrome $c$ peroxidase association. Science 241:67-70. doi:10.1126/science.2838904

Pelletier H, Kraut J (1992) Crystal-structure of a complex between electron-transfer partners, cytochrome $c$ peroxidase and cytochrome $c$. Science 258:1748-1755. doi:10.1126/science.1334573

Pervushin K, Riek R, Wider G, Wüthrich K (1997) Attenuated T2 relaxation by mutual cancellation of dipole-dipole coupling and chemical shift anisotropy indicates an avenue to NMR structures of very large biological macromolecules in solution. Proc Natl Acad Sci 94:12366-12371

Pollock WB, Rosell FI, Twitchett MB, Dumont ME, Mauk AG (1998) Bacterial expression of a mitochondrial cytochrome $c$. Trimethylation of lys72 in yeast iso-1-cytochrome $c$ and the alkaline conformational transition. Biochemistry 37:6124-6131. doi:10. 1021/bi972188d

Scanu S, Forster J, Finiguerra MG, Shabestari MH, Huber M, Ubbink M (2012) The complex of cytochrome $f$ and plastocyanin from Nostoc sp PCC 7119 is highly dynamic. ChemBioChem 13:1312-1318. doi:10.1002/cbic.201200073

Schilder J, Ubbink M (2013) Formation of transient protein complexes. Curr Opin Struct Biol 23:911-918. doi:10.1016/j. sbi.2013.07.009

Schilder J, Lohr F, Schwalbe H, Ubbink M (2014) The cytochrome c peroxidase and cytochrome c encounter complex: the other side of the story. FEBS Lett 588:1873-1878. doi:10.1016/j.febslet.2014.03.055

Schilder J, Liu WD, Kumar P, Overhand M, Huber M, Ubbink M (2015) Protein docking using an ensemble of spin labels optimized by intramolecular paramagnetic relaxation enhancement. Phys Chem Chem Phys 18:5729-5742. doi:10.1039/c5cp03781f

Schwieters CD, Kuszewski JJ, Tjandra N, Clore GM (2003) The Xplor-NIH NMR molecular structure determination package. J Magn Reson 160:65-73. doi:10.1016/S1090-7807(02)00014-9

Schwieters CD, Kuszewski JJ, Clore GM (2006) Using Xplor-NIH for NMR molecular structure determination. Prog Nucl Mag Res Spectrosc 48:47-62. doi:10.1016/j.pnmrs.2005.10.001

Shortle D, Ackerman MS (2001) Persistence of native-like topology in a denatured protein in $8 \mathrm{M}$ urea. Science 293:487-489. doi:10. 1126/science. 1060438

Suh JY, Tang C, Clore GM (2007) Role of electrostatic interactions in transient encounter complexes in protein-protein association investigated by paramagnetic relaxation enhancement. J Am Chem Soc 129:12954-12955. doi:10.1021/ja0760978

Tang C, Iwahara J, Clore GM (2006) Visualization of transient encounter complexes in protein-protein association. Nature 444:383-386. doi:10.1038/nature05201 
Tang C, Schwieters CD, Clore GM (2007) Open-to-closed transition in apo maltose-binding protein observed by paramagnetic NMR. Nature 449:1078-1082. doi:10.1038/nature06232

Tang C, Ghirlando R, Clore GM (2008a) Visualization of transient ultra-weak protein self-association in solution using paramagnetic relaxation enhancement. J Am Chem Soc 130:4048-4056. doi: $10.1021 / \mathrm{ja} 710493 \mathrm{~m}$

Tang C, Louis JM, Aniana A, Suh JY, Clore GM (2008b) Visualizing transient events in amino-terminal autoprocessing of HIV-1 protease. Nature 455:693-696. doi:10.1038/nature07342

Ubbink M (2009) The courtship of proteins: understanding the encounter complex. FEBS Lett 583:1060-1066. doi:10.1016/j. febslet.2009.02.046

Ulucan O, Helms V (2015) How hydrophilic proteins form nonspecific complexes. J Phys Chem B 119:10524-10530. doi:10. 1021/acs.jpcb.5b05831

Van de Water K, Van Nuland NAJ, Volkov AN (2014) Transient protein encounters characterized by paramagnetic NMR. Chem Sci 5:4227-4236. doi:10.1039/C4sc01232a

Vaynberg J, Qin J (2006) Weak protein-protein interactions as probed by NMR spectroscopy. Trends Biotechnol 24:22-27. doi:10. 1016/j.tibtech.2005.09.006

Villareal VA, Spirig T, Robson SA, Liu MY, Lei BF, Clubb RT (2011) Transient weak protein-protein complexes transfer heme across the cell wall of Staphylococcus aureus. J Am Chem Soc 133:14176-14179. doi:10.1021/ja203805b

Volkov AN, Worrall JA, Holtzmann E, Ubbink M (2006) Solution structure and dynamics of the complex between cytochrome $c$ and cytochrome $c$ peroxidase determined by paramagnetic NMR. Proc Natl Acad Sci USA 103:18945-18950. doi:10.1073/ pnas.0603551103

Volkov AN, Bashir Q, Worrall JAR, Ubbink M (2009) Binding hot spot in the weak protein complex of physiological redox partners yeast cytochrome $c$ and cytochrome $c$ peroxidase. J Mol Biol 385:1003-1013. doi:10.1016/j.jmb.2008.10.091

Volkov AN, Bashir Q, Worrall JA, Ullmann GM, Ubbink M (2010a) Shifting the equilibrium between the encounter state and the specific form of a protein complex by interfacial point mutations. J Am Chem Soc 132:11487-11495. doi:10.1021/ja100867c

Volkov AN, Ubbink M, van Nuland NA (2010b) Mapping the encounter state of a transient protein complex by PRE NMR spectroscopy. J Biomol NMR 48:225-236. doi:10.1007/s10858$010-9452-6$

Volkov AN, Nicholls P, Worrall JAR (2011) The complex of cytochrome $c$ and cytochrome $c$ peroxidase: the end of the road? BBA-Bioenerg 1807:1482-1503. doi:10.1016/j.bbabio.2011.07. 010

Vranken WF et al (2005) The CCPN data model for NMR spectroscopy: development of a software pipeline. Proteins 59:687-696. doi:10.1002/prot.20449

Worrall JA, Kolczak U, Canters GW, Ubbink M (2001) Interaction of yeast iso-1-cytochrome $c$ with cytochrome $c$ peroxidase investigated by $\left[{ }^{15} \mathrm{~N},{ }^{1} \mathrm{H}\right]$ heteronuclear NMR spectroscopy. Biochemistry 40:7069-7076. doi:10.1021/bi0025823

Xing Q et al (2014) Visualizing an ultra-weak protein-protein interaction in phosphorylation signaling. Angew Chem Int Ed Engl 53:11501-11505. doi:10.1002/anie.201405976

Xu X, Reinle W, Hannemann F, Konarev PV, Svergun DI, Bernhardt R, Ubbink M (2008) Dynamics in a pure encounter complex of two proteins studied by solution scattering and paramagnetic NMR spectroscopy. J Am Chem Soc 130:6395-6403. doi:10. 1021/ja7101357

Xu X, Keizers PH, Reinle W, Hannemann F, Bernhardt R, Ubbink M (2009) Intermolecular dynamics studied by paramagnetic tagging. J Biomol NMR 43:247-254. doi:10.1007/s10858-0099308-0

Yonetani T (1965) Studies on cytochrome $c$ peroxidase. II. Stoichiometry between enzyme, $\mathrm{H}_{2} \mathrm{O}_{2}$, and ferrocytochrome $c$ and enzymic determination of extinction coefficients of cytochrome c. J Biol Chem 240:4509-4514

Zlotnick A (2005) Theoretical aspects of virus capsid assembly. J Mol Recognit 18:479-490. doi:10.1002/jmr.754 